\title{
El Informe Brandt y el Comercio de productos industrializados
}

\author{
INTRODUCCIÓN
}

\section{1) Problemas observados en el comercio de manufacturas del Sur}

Como paso previo a discutir las recomendaciones de la Comisión. Brandt en materia de comercio de productos industriales, se requiere señalar los problemas centrales observados hasta el presente y analizar someramente sus causas inmediatas ${ }^{1}$. Tales problemas, que por lo demás no son independientes, pueden resumirse así:

A) Los bienes manufacturados en que se han concentrado las exportaciones del Sur al Norte han resultado sometidos a varios de los problemas que tradicionalmente se adscribían a la exportación de productos básicos ${ }^{2}$, a saber: bajo incremento de la demanda interna en los países del Norte ${ }^{3}$; creciente proteccionismo a su producción local, que dificulta el crecimiento de las exportaciones del Sur a expensas de ésta; bajos márgenes de ganancia y tendencia al deterioro de los precios relativos, debido a una alta concurrencia en la oferta frente al bajo crecimiento de la demanda y apropiación por parte de las corporaciones transnacionales (CIN) de una parte grande y creciente de las ganancias potenciales en el comercio.

La concentración en bienes con estas características está fundamentalmente ligada al desigual desarrollo tecnológico y a la inexistencia o imperfecciones en los mercados de tecnología ${ }^{4}$. Ello impide, dificulta o encarece a los países del Sur el desarrollo de los sectores más dinámicos o los obliga a recurrir para ello a las CiN. Al mismo tiempo ello determina las tendencias en precios relativos en el comercio de manufacturas.

En segundo lugar, influye la política comercial de los países del Norte, y en particular sus estructuras arancelarias, las cuales otorgan

\footnotetext{
${ }^{1}$ El Informe no presenta un diagnóstico ni un análisis completo de tal problemática, si bien en varias partes recoje, explícita o implícitamente, algunos elementos.

${ }^{2}$ Ver Keesing (1976). El Informe presta una mínima atención a esta problemática general.

${ }^{3}$ Los principales rubros (textiles, confecciones, calzado, alimentos procesados) sufren, al igual que muchos productos básicos de consumo masivo, la ley de Engel. (1978).

${ }^{4}$ Sobre la determinación "tecnológica" de los flujos de comercio, ver Harry Johnson
} 
una protección efectiva creciente con el grado de procesamiento de los bienes ${ }^{5}$. Estas políticas afectan en especial el desarrollo de exportaciones de productos básicos procesados por parte de los países del Sur.

En tercer lugar, influye la propia política comercial y de desarrollo de los países del Sur, la cual en general no le ha prestado la atención debida al problema del desarrollo tecnológico y de la apropiación de las ganancias potenciales del comercio por parte de las CIN.

B) El proteccionismo creciente en los países del Norte recibe una gran atención en el Informe. Este no destaca, sin embargo, a mi parecer, un aspecto crucial, el hecho de que éste proteccionismo es discriminatorio contra los países del Sur. Ello se debe antetodo al menor poder de negociación y retaliación de estos países ${ }^{6}$. La mayor parte de las medidas proteccionistas, como el mismo Informe lo reconoce ${ }^{7}$ han sido violatorias del GATT y sucede que este no comtempla sanciones distintas al derecho de represalia para la parte afectada. Aún más, el caso más protuberante de proteccionismo discriminatorio contra el Sur ha sido legitimado por el GATT mediante el Acuerdo Multifibras, en violación flagrante de su propio texto, por cuanto el Acuerdo es discriminatorio, contiene restricciones cuantitativas permanentes, permite crear nuevas barreras sin demostrar la realidad o la amenaza de "daño grave" a la producción nacional y ni siquiera autoriza a adoptar represalias.

Ello no es sino reflejo de la desigual capacidad de negociación y represalia, frente a las cuales no se ha hecho absolutamente nada a nivel internacional.

De otra parte, las exportaciones de manufacturas por parte del Sur se han concentrado en un puñado de países, con lo cual los 'latecomers' sufren con especial rigor los efectos de la concurrencia y el proteccionismo provocado ante el éxito de los primeros ${ }^{8}$. Ello, por demás, ha creado una gran diferenciación de intereses entre los países del Sur, lo cual dificulta las posiciones conjuntas en las mesas de negociación y reduce su poder relativo en ellas.

G) Una proporción grande y creciente del comercio en productos industriales, y de las exportaciones del Sur, en particular, se encuentra

${ }^{5}$ Ver BIRF (1977a) y (1977b).

${ }^{6}$ Ver G. Perry (1979).

${ }^{7}$ Pág. 180.

${ }^{8}$ Algunos mecanismos de protección tienen incluso el efecto de "congelar" las flujos de comercio y beneficiar a los "primeros". 
dominada por las ciN's, bien sea como transacciones intrafirma o a través de diversas formas de subcontratación". El informe Brandt registra este hecho pero no señala el problema: una alta proporción de las ganancias potenciales del comercio son apropiadas por las $\operatorname{CIN}^{\prime} \mathrm{s}^{10}, \mathrm{y}$ remitidas a su país de origen ${ }^{11}$. El valor agregado que queda en el país puede llegar a reducirse a los pagos por salarios.

La internalización del comercio en las CrN's, como vinos, está íntimamente vinculado al desigual desarrollo tecnológico (Sur-Norte) y la inexistencia o imperfección de los mercados tecnológicos. Pero además, se ha visto reforzada por: la virtual inexistencia entre 1950 y 1970 de un mercado libre de capitales, lo cual obligaba a los países del Sur a recurrir o bien a la 'ayuda externa', con sus muchos condicionantes, o a las CTN's para satisfacer sus necesidàdes de inversión y balanza de pagos $^{12}$; la estructura oligapolística de los mercados internos en el Norte, que exige con frecuencia recurrir a las CTN para tener acceso a ellos; la política comercial de los países del Norte: regímenes arancelarios preferenciales para las operaciones de subcontratación ${ }^{13}$; inclusión preferencial en los Sistemas Generales de Preferencias (SGP) de productos que fabrican sus propias cin en el Sur; y por la política comercial de los países del Sur que ha favorecido las exportaciones de manufacturas a través de los CiN, mediante mecanismos tales como las zonas francas, los sistemas de 'drawback', etc.

D) El comercio de manufacturas del Sur se ha concentrado excesivamente en el Norte. Si bien ha habido un gran desarrollo del intercambio intrarregional en el Sur, el Comercio Inter-regional Sur-Sur y con los países socialistas es apenas incipiente.

Este fenómeno se debe básicamente al desarrollo, histórico de lả infraestructura comercial (transporte, comunicaciones, servicios financieros y de mercado, etc.) en la dirección Centro-Periferia. Pero ha sido reforzado por:

${ }^{9}$ Para una descripción cuantitativa de este fenómeno. Ver Helleiner (1977), Finger (1975), Shonfield (1976) y Perry (1979).

${ }^{10}$ Los resultados tradicionales de la teoría del comercio internacional se modifican sustancialmente al introducir la consideración de este fenómeno. Ver por ejemplo Bhagwati y Tironi.

${ }^{19}$ Son múltiples las formas como las cin's pueden apropiarse de y girar una proporción mayor de las utilidades de las que corresponde a su aporte de capital y a las regulaciones locales: subfacturación de exportaciones; sobre-facturación de importaciones y pagos por servicios (tecnología, administración, financiación) a empresas filiales.

${ }^{12} \mathrm{C}$. Díaz Alejandro (1975) señala que los paises del Norte estimularon preferencialmente la exportación de capitales en la forma de inversión directa.

${ }^{13} \mathrm{Ver}$, por ejemplo, Finger (1975). 
- el rol central y creciente de las CTN en las exportaciones de manufacturas del sur;

- los criterios de fijación de tarifas en las convenciones internaciones de transporte ${ }^{14}$;

— las condiciones de la 'ayuda externa' que dificultan la adquisición de bienes y equipos en otros países del Sur o en países socialistas;

- la ausencia de negociaciones de desgravación Sur-Sur en el seno del GATT;

- la política comercial de los países del Sur que ha carecido de la voluntad y agresividad requerida para superar estos problemas.

\section{2) La Discusión de las Recomendaciones de la Comisión}

Aun cuando algunas recomendaciones concretas de la Comisión demuestran su sensibilidad a todos los problemas discutidos arriba, en general ellas se centran en buscar un mayor crecimiento de las exportaciones industriales del Sur, sin atender suficientemente al problema de su composición y conveniencia o al rol de las CTN. Por ello su análisis y recomendaciones enfocan fundamentalmente el problema de proteccionismo en el Norte y de la requerida expansión del comercio SurSur. Esta óptica naturalmente se adapta a la tónica general del Informe; su 'realismo político' centrado en la búsqueda de ganancias mutuas y de persuasión al Norte, en este caso en el sentido de que la ampliación del comercio internacional le beneficia. La consideración de los problemas señalados arriba en A) y C); y algunos relacionados con el proteccionismo mismo (la naturaleza misma del Gatt y sus' negociaciones), inevitablemente llevan a encarar la existencia de conflicto de intereses y poder desigual; por ello no es sorprendente que se les preste menos atención en el Informe y que no se señale su naturaleza conflictiva. Si bien la aproximación pragmática de la Comisión puede permitir avanzar más en las negociaciones internacionales a corto plazo, frente a las posiciones maximalistas de algunos grupos, es importante que se tengan plena conciencia de las grandes limitaciones de su enfoque y sus recomendaciones ante la aborda.

Por ello he preferido plantear un conjunto de recomendaciones que se deriva del análisis previo, en lugar de partir de la discusión contenida en el Informe, desarrollando con mayor detalle las propuestas que difieren de las de la Comisión. Las propuestas se agrupan así:

\footnotetext{
${ }^{14}$ Finger (1976). Estas además, contribuyen a incrementar artificialmente la protección efectiva al procesamiento de productos primarios en el Norte.
} 
1. Relacionadas con el Gatt (Sección II);

2. Con otras instituciones internacionales (Sección III);

3. Con medidas conjuntas entre países del Sur y entre estos y los países socialistas (Sección InI);

4. Con la política interna en los países del Sur (Sección v).

1. Medidas Propuestas en relación con el comercio de manufacturas Sur-Norte.

a) Reducciones arancelarias.

Lo más conveniente para los intereses de los PED como conjunto sería la reducción de las tarifas NMF y su dispersión en los países industrializados (PI) sobre la totalidad del universo arancelario, sin concesiones recíprocas equivalentes por parte de lọ PED. La aplicación generalizada de una fórmula automática semejante traería algunas de las ventajas siguientes y evitaría exclusión de productos "sensitivos" para los PI; vale decir, los de mayor interés para los PED; como ha venido ocurriendo. Minimizaría los efectos actuales del "escalonamiento" de la estructura arancelaria en los PI en contra del procesamiento de materias primas en los PED. Evitaría las inconveniencias derivadas del carácter discrecional y concesional de los SGP (exclusión de productos "sensitivos", cuotas limitativas, inclusión preferencial de bienes producidos en los PED por las CTN; manipulación política) a tiempo que se obtendrían esencialmente las mismas ventajas, puesto que los SGP han tenido mayor efecto sobre creación que sobre desviación de comercio $^{15}$. Sin embargo, la reducción de márgenes de preferencia sería inconveniente para los países más pobres ${ }^{16}$, los cuales habrían de ser compensados en otra forma. Reduciría la tendencia a la consolidación -de relaciones comerciales neocoloniales y la congelación de flujos de comercio Norte-Sur por "bloques regionales" (Europa-Africa, Japón-ASEAN, EE. UU.-A.L.) implícita en acuerdos como el de Lomé y la aplicación misma de los SGP. La erosión de preferencias regionales, sin embargo, se enfrentaría a la oposición de los ACP firmantes de la Convención de Lomé. Evitaría Ios problemas asociados con "acuerdos sectoriales especiales", como el del Multifibras y el impulso a su proliferación por parte de algunos PI.

${ }^{15}$ Véase por ejemplo Cline (1977) e IBRF (1977 a 1977b).

${ }^{15}$ Ibid. 
b) Reducción o eliminación de las barreras no-arancelarias en los PI, sin concesiones reciprocas equivalentes por parte de loS $P E D$.

Esta medida, complementaría a la anterior, exigiría ante todo la eliminación o revisión drástica del Acuerdo Multifibras. Se ha calculado que la liberación del comercio de textiles y confecciones tendría a mediano plazo un impacto sobre el valor de las exportaciones de los PED a los PI, superior a la suma del impacto de una liberación total del comercio en otros productos manufacturados y en productos básicos ${ }^{17}$.

c) Formulación y Aplicación más estricta de cláusulas de salvaguardia.

El informe hace suficiente énfasis en este punto.

d) Institución y Aplicación de un Sistema Efectivo de Sanciones por Violación al Acuerdo.

El punto anterior sin embargo, no tendría ninguna efectividad sin resolver este último problema. Como el mismo informe admite "muchos países han invocado el artículo 19 (clásulas de salvaguardia), pero con mayor frecuencia han levantado barreras no-arencelarias violando tal artículo"18.

De hecho, mientras el GATT no se complemente con efectivas instituciones jurídicas supranacionales o su texto no se adopte como ley nacional en todos los países industrializados, por más que se precise y restrinja en la letra la utilización de las cláusulas de excepción, éstas seguirán siendo aplicada en forma discriminatoria contra los PED que no posean poder suficiente para adoptar represalias.

Más aún, en el seno mismo del GA'Tr se suscribió el Acuerdo textilero, que contradice de sus principios.

Una institución supranacional evitaría una violación tan flagrante del texto básico del Acuerdo.

\section{Medidas propuestas en relación con el Comercio Sur-Sur.}

La medida más importante a este respecto sería la de autorizar sin limitaciones la adopción de concesiones unilaterales, bilaterales o multilaterales, entre los países del Sur, sin que ellas se transmitan automáticamente a los países del Norte por el imperio de la cláusula de NMF. El Acuerdo ya contempla excepciones a esta cláusula respecto de los

${ }^{17}$ Cline (1977) e IBRF (1977a y b).

${ }^{18}$ Ibíd. p. 180. 
acuerdos de integración económica o áreas aduaneras de libre comercio.

\section{Problemas}

La adopción de estas medidas se enfrenta a dos problemas básicos:

1. Implicaría una modificación profunda del espíritu implícito del GATT, que los PI no estarían en disponibilidad de aceptar. En efecto, las negociaciones en el GATT se han planteado básicamente entre quienes se hacen concesiones recíprocas, en un plano de igualdad, lo cual virtualmente ha excluído a los países en desarrollo. En esas condiciones no es de extrañar que las menores rebajas hayan ocurrido en productos en los cuales aquellos son muy competitivos, ni que en las naciones industrializadas se haya mantenido una estructura arancelaria que desestimula el procesamiento de materias primas en los países en desarrollo.

El GATT ha sido ante todo un instrumento para la ampliación del comercio Norte-Norte. De allí que los PED hubiesen recurrido a otro organismo, la UNCTAD,-para plantear sus reivindicaciones; en particular, la concesión de ventajas, no recíprocas, absolutas, permanentes y generalizadas (UNCTAD 1, 1964). Los PI aceptaron este principio en el GATT en 1966, pero le han dado un desarrollo muy parcial y desfigurado con los SGP (concesiones parciales, temporales, discrecionales, excluyentes y limitadas). Una avenida de acción para los PED sería la de buscar modificar la naturaleza de los SGP en la dirección de su petición originaria. La otra sería la acá expuesta, en la cual ia "ventaja" para los PED consistiría en beneficiarse de reducciones generalizadas sin ofrecer concesiones recíprocas equivalentes. Este último principio ha sido reconocido por el GATT. Pero su aplicación en la forma aquí sugerida, exigiría un cambio fundamental en el sistema de negociaciones.

El mayor cambio consistiría en la creación de una institución supranacional con poder real para aplicar sanciones efectivas a la violación del Acuerdo. La "filosofía" implícita del GATT ha sido la de negociaciones entre iguales, con poder de represalia similar ante violaciones de los acuerdos.

Vale decir, habría que transformar (o sustituir) unas instituciones creadas por el Norte para su propio beneficio (y que han "beneficiado" al Sur apenas por un efecto secundario de "spill-over") en (o por) unas que "acomoden todos los intereses"; como lo sugiere el mismo Informe al reconocer que a largo plazo se requiere una institucionalidad única que absorba al GATT y la UNCTAD y que mientras tanto, estas continuarían expresando los intereses diferentes que de hecho expresan. 
2. Implicaría un acuerdo entre los PED respecto de sus intereses comunes de largo plazo. Tal acuerdo lo dificulta la existencia misma de la Convención de Lomé y los SGP, que han creado intereses regionales o individuales diferenciados. Las propuestas acá consignadas tendrían, además de las mencionadas, la ventaja de reducir esta fisuras en la posición negociadora conjunta de los PED hacia el futuro.

\section{OTRAS MEDIDAS A NIVEL INTERNACIONAL}

Los problemas observados en la evolución del comercio de manufacturas (ver Anexo) y el análisis de sus causas, sugieren que ciertas medidas en otros frentes podrían tener una mayor.incidencia en la evolución a mediano plazo del comercio en manufacturas que las propias medidas comerciales. Ellas se refieren a 1) una mayor liberalización del mercado internacional de capitales y, especialmente, del de tecnología y a 2) un mayor apoyo financiero y técnico a lOS PED para promover un desarrollo tecnológico "self-reliant"19; mejorar su capacidad negociadora en el mercado de tecnología y frente a las cin; crear y mejorar capacidad de comercialización internacional y, desarrollar la infraestructura (transporte, comunicaciones, servicios financieros y de mercado) para el intercambio Sur-Sur.

Otras medidas serían modificaciones en los criterios de fijación de fletes en las Convenciones Internacionales de Transporte, etc.

A estos fines podrían coayudar medidas como los Códigos de Transferencia de Tecnología y Conducta de los Cin; las instituciones multinacionales con estos fines específicos y la acción de otras en estos frentes; y otras, que serán objeto de discusión en otras sesiones de este Encuentro. Queremos acá relevar una propuesta que vincula la solución en forma directa al desequilibrio comercial en productos industriales intensivos en tecnología, entre Sur y Norte. Dicha propuesta ${ }^{20}$ sugiere establecer un gravámen proporcional al saldo en este tipo de comercio, cuyo recaudo se transfiera automáticamente a Fondos Regionales bajo el control exclusivo de los $P E D$, los cuales los utilizarían con los objetivos mencionados arriba en 2).

\footnotetext{
${ }^{19}$ Sobre este concepto ver Sagasti.

${ }^{20}$ Sagasti, Pizano y Perry (1979).
} 
A. Acciones Conjuntas entre Países del Sur

El informe trae un catálogo muy completo de recomendaciones respecto a la cooperación entre países del Sur (p. 133-140). Acá simplemente los reordenaremos de acuerdo con el análisis de la Introducción.

1) Medidas para Enfrentar los Desequilibrios en Comercio de Bienes Intensivos en Tecnología y el Predominio de las CIN's en el Comercio.

- Cooperación para un desarrollo tecnológico "self-reliant".

- Cooperación en la comercialización internacional.

- Creación y promoción de empresas productoras y comercializadoras entre países del Sur.

2. Otras medidas para el Desarrollo del Comercio Sur-Sur. Además de las anteriores.

- Cooperación para el desarrollo de la infraestructura comercial (transporte, comunicaciones, servicios financieros y de mercadeo).

- Acuerdos de comercio y coproducción multilaterales regionales (integración económica, mercados comunes, áreas de libre comercio); acuerdos por sectores (v. gr. para el procesamiento de productos básicos) y bilaterales, especialmente entre países vecinos o con disponibilidad común de ciertos recursos naturales.

3) Desarrollo de posiciones y procedimientos de negociación conjunta y retaliación, ante violaciones de los Acuerdos, frente al Norte.

B. Otras Medidas para el Desarrollo del Intercambio Sur-Países Socialistas.

El tratamiento del Informe sobre este tema es muy superficial y se limita a recomendar que los países socialistas efectúen una mayor parte de su intercambio en monedas convertibles, lo cual tendría una importancia absolutamente secundaria y desconoce las razones por las cuales se da una tendencia en los PS al equilibrio bilateral y el déficit en monedas convertibles ${ }^{21}$. Las medidas conjuntas que podrían contribuir más a este desarrollo, son las siguientes: acuerdos especiales con el CAME y el BII; Cooperación para el Desarrollo de la infraestructura comercial; acuerdos comerciales, de coproducción, intercambio tecno-

${ }^{20}$ Sagasti ( ), Pizano y Perry' (1979).

${ }^{21}$ Ver G. Perry (1978). 
lógico y financiamiento; creación y promoción de empresas mixtas de producción y comercialización; acuerdos especiales sobre compras públicas y desarrollo tecnológico en sectores estatales.

\section{POLITICA COMERCIAL Y DE DESARROLLO EN LOS PED.}

\section{A. Politica Comercial de Industrialización.}

Ante todo se debe superar el falso dilema entre políticas de sustitución de importaciones y promoción de exportaciones que se ha venido popularizando y que recoge el Informe (p. 173-174) sin adoptar una posición crítica. El apoyo de la política comercial al desarrollo de la industrialización debe inscribirse en un contexto de planeación global en el cual el rol central corresponde al desarrollo de la capacidad tecnológica, al proceso de acumulación nativo, a la ampliación de los mercados internos y al desarrollo de la infraestructura industrial. La política comercial, en este contexto, debe apoyar los demás instrumentos de política para promover el desarrollo de sectores que presenten un alto valor agregado nacional, directo e indirecto (v. gr. intensivos en recursos naturales y producción local; con mercados suficientes para aprovechar economías de escala potenciales), externalidades económicas (v. gr. intensivos en mano de obra abundante, etc.) y externalidades tecnológicas por capacitación de la mano de obra, dominio de procesos utilizables en otros sectores, incidencia en el progreso técnico de otros sectores (v. gr. en el caso de la industria de bienes de capital) ${ }^{22}$.

Para ello, los sectores con estas características deben obtener una adecuada protección efectiva (generalmente en un contexto de planeación dinámica) tanto para sus ventas domésticas como externas (mediante el uso coordinado de la tasa de cambio, los aranceles e instrumentos paraarancelarios y los subsidios a la exportación), de modo que no se creen discriminaciones artificiales entre la producción doméstica y la exportación. Los errores en la política de "sustitución de importaciones" ad extremum practicada por algunos países se centraron en: sur carácter generalizado y no selectivo; permanente y no temporal; a favor de sectores que no cumplían las condiciones enumeradas; discriminatorio

${ }^{22}$ De paso, el informe parece considerar equivocadamente, que solamente los países grandes pueden desarrollar esta industria: en la mayoría de los casos no se presentan grandes economias de escala en esta industria; sus procesos no son muy sofisticados tecnológicamente y ocupan mucha mano de obra. El informe se abstiene de señalar estas y otras ventajas (la incidencia en el progreso técnico de los sectores a que se dirigen) del desarrollo de esta industria. 
contra las exportaciones y excesivamente a favor del capital extranjero. Algunos de estos errores, o su inversa, se están cometiendo en la "promoción de exportaciones": carácter generalizado; permanente; favoreciendo sectores o fórmulas que dejan un bajo valor agregado nacional, mediante el uso de esquemas tales como zonas francas, sistemas de "drawback" y subsidios proporcionales al valor bruto de la producción o de sus costos (como en el caso del crédito); discriminando contra las ventas domésticas; favoreciendo excesivamente al capital extranjero.

En segundo término, la política comercial debe desarrollar la capacidad propia de "comercialización internacional".

En tercer lugar debe buscar una mayor diversificación regional, introduciendo unilateralmente medidas específicas que enfrenten algunos problemas especiales para el desarrollo del intercambio con otros países del Sur y con Países Socialistas.

Por último, debe contemplar la posibilidad de compensar algunos efectos de la política discriminatoria del Norte (v. gr. prohibiendo o gravando la exportación de algunos productos básicos no procesados; identificando y utilizando instancias efectivas de retaliación, etc.).

\section{BIBLIOGRAFIA}

Balassa, B .: "Tariff Protection in Industrial Countries an Evaluation", Journal of Political Economy, Dec. 1965.

Baldwin y Murray, "MFN tariff reductions and developing countries trade benefits under the GSP". Economic journal, March, 1977.

Cline, W., et. al.: Multilateral Effect or Tariff Negotiations in the Tokio Round, mimeo, 1977.

C.P.U. América Latina y el Nuevo Orden Económico Internacional. Eduardo Hill-Luciano Tomassini, ed. 1979.

Díaz-Alejandro, Carlos: "North-South Relations: The Economic Component" World Politics and International Economics; Bergsten, F. y Kraiuse L., ed. Brookings Institution, 1975.

Finger, J.M. "Effects of the Kennedy Round Concessions on the Exports of Developing Countries" Economic Journal, June 1975.

- "Tariff Provisions for Offshore Assembly and the Export of Developing Countries", Economic Journal, June, 1975.

- "Effective protection by transportation cost and tariffs" Q.J.E. Feb. 1976.

Helleiner, G.K.: "Structural Aspects of Third World Trade", ISS 25th Aniversary Conference, 1977. 
- "Manufactured Exports from Less Developed Countries and Multinational firms", Economic Journal, 83 March 1973.

- "International Enterprises and the New Political Economy of US Trade Policy", Oxford Economics Papers, 19 March 1977.

- "Intrafirm Trade and the Developing Countries: Patterns, Trends, and Data Problems", Seminar of Intrafirm Transactions, UNCTAD y I SD Sussex, Nov. 1977.

IBRF (Banco Mundial' "Propects for Developing Countries", 1977. "Trade Liberalization and Export Promotion", June 1977.

International Trade Center, UNCTAD-GATr: "Structural and Cyclical Changes in International Trade, and their Impact on the export performance and prospects of Developing Countries". Ginebra, enero 1978.

Lobal, Z.: "Trade Effects on the Generalized systems of Preferences", IMF, april 1975.

Keesing, D.: "Industrial Countries Manufactured Imports from Developing Countries, Recent Trends and Prospects", sid Conference, Amsterdam, 1976.

- "Recent Trends in Manufactured and Total Exports from Developing Countries", mimeo, June. 1977.

Perry, G.: "Relaciones Económicas de Colombia con países Socialistas", Coyuntura Económica, FEDESARROLLO, agosto 1977.

Shonfield, A. et. al. "International Dialogue on Commodities: Resources Policy, June 1976.

UNCTAD. "Evolución y Tendencias recientes del Comercio de Manufacturas y Semimanufacturas", TD/B/C. 2./75, mayo 1977.

Wolter F. "Non tariff Barriers and the Export perforance of Developing Economics. 